\title{
Semblanza de la doctora Patricia Camarena Homenaje póstumo
}

\author{
María Trigueros Gaisman ${ }^{1}$
}

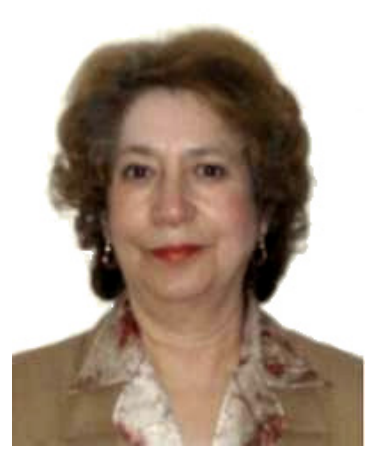

La noticia reciente de la pérdida de la doctora Patricia Camarena Gallardo me sorprendió por inesperada. Hacía tiempo que no la veía, aunque nos manteníamos en contacto no muy frecuente, pero con gran cariño.

Conocí a Paty más de cerca cuando organizamos juntas una sección sobre Enseñanza del Cálculo Diferencial e Integral en el XXX Congreso de la Sociedad Matemática Mexicana en la Universidad Autónoma de Aguascalientes en 1997. La experiencia de trabajar con ella fue sumamente grata. La descubrí como una persona sencilla, gentil y muy responsable. A raíz de esa etapa de trabajo intenso conjunto seguimos viéndonos para comer o tomar un café con cierta frecuencia, cuando sus múltiples responsabilidades académicas se lo permitían.

Para quienes nos interesamos en el estudio de la Educación Matemática la noticia fue desoladora. Todos la recordaremos como siempre fue: sencilla, alegre, amable, responsable, dinámica, cálida y carismática. La recordaremos también por su contribución a la Educación Matemática a través de dos desarrollos importantes, por un lado, la creación y el uso de la Teoría de la Matemática en el Contexto de las Ciencias en la que trabajó durante más de treinta años y que es conocida y reconocida en toda Latinoamérica y otras partes del mundo. Por

\footnotetext{
1 tigre@itam.mx
} 
otro lado, por el desarrollo de la Matemática Social en México. La propia doctora Camarena describe:

La teoría educativa de la Matemática en el Contexto de las Ciencias nació en 1982, en el Instituto Politécnico Nacional (IPN) de México; se enfoca en las carreras universitarias donde la matemática no es una meta en sí misma, es decir, donde no se van a formar matemáticos... y reflexiona acerca del vínculo entre la matemática y otras ciencias, situaciones profesionales, laborales y actividades de la vida cotidiana. (Camarena, 2013, p. 21).

Por otra parte, y para poner en práctica los elementos de esta teoría, desarrolló la línea de investigación conocida como Matemática Social, la cual, en sus palabras:

[...] pretende que el profesor de matemáticas contribuya con su práctica docente a la formación integral del futuro profesionista... incidiendo en la interdisciplinariedad dentro del ambiente de aprendizaje que hace reflexionar sobre las matemáticas para qué, por qué dar matemáticas, qué dar de matemáticas. Se desarrolla por medio de la investigación científica, en la línea de investigación denominada Matemática Social. Con ella se pretende que el profesor de matemáticas contribuya con su práctica docente a la formación integral del futuro profesionista... así como en su vida cotidiana. (Camarena, 2013, p. 21).

Patricia Camarena reconoció muy tempranamente la necesidad de estudiar el aprendizaje y la enseñanza de las matemáticas en el contexto de las ciencias en el nivel universitario, incluyendo la modelación como parte de la enseñanza de las matemáticas. Esta idea fue después aplicada al contexto de la ingeniería, la biología y la economía y fue también adaptada a otros niveles escolares. Recientemente el interés y la investigación en esta área ha ido en aumento. Paty Camarena debe reconocerse como la pionera. Insistió siempre en que el trabajo en equipo entre los alumnos era esencial para desarrollar las habilidades necesarias en el trabajo profesional y en demostrar que el papel de las matemáticas en la vida cotidiana era indispensable en la formación de los estudiantes.

Patricia fue la primera mujer egresada de la licenciatura en Matemáticas de la Escuela Superior de Física y Matemáticas (ESIME) del Instituto Politécnico Nacional (IPN). Desde sus estudios profesionales permaneció ligada a esta 
institución en la que forjó su trayectoria profesional y en la que inició la línea de investigación en Matemática Social. En ella ocupó distintos puestos académicos y administrativos y en los últimos años como profesora e investigadora de tiempo completo en la Escuela Superior de Ingeniería Mecánica y Eléctrica (ESIME). Realizó sus estudios de doctorado en Matemática Educativa en el departamento del mismo nombre del CINVESTAV. En su trabajo de tesis elaboró cuidadosamente la teoría de la Matemática en el contexto de las ciencias que utilizó desde ese momento en todos sus trabajos de investigación. Para ella, la investigación y la docencia formaban, junto con el cuidado de su familia, su proyecto de vida.

Durante toda su vida profesional, Patricia, realizó un trabajo de liderazgo e hizo una labor constante y consistente en diversos foros nacionales e internacionales que hizo posible congregar a la comunidad de científicos interesados en la comprensión de la enseñanza y el aprendizaje de las matemáticas en distintas instituciones y en diferentes niveles de educación.

El trabajo de la doctora Camarena ha sido reconocido tanto en su institución como fuera de ella. Obtuvo el Premio a la Investigación del IPN en el área de Educación en 1990. Fue reconocida también con el primer lugar en el Premio a la mejor Tesis de doctorado en Investigación Básica en Educación Matemática otorgado por la Sociedad de Científicos del Centro de Investigación y Estudios Avanzados (CINVESTAV) en 1999, además del Premio a la mejor tesis de doctorado de la Asociación Nacional de Universidades e Instituciones de Educación Superior de México (ANUIES) en el año 2000. En el 2014 fue galardonada con el Premio Coatlicue que otorga la Coordinación Internacional de Mujeres en el Arte, como reconocimiento de su dedicación a la ciencia y por enaltecer con su obra la cultura universal. Desde 2003 fue miembro del Comité Interamericano de Educación Matemática (CIAEM) como su representante en México. Colaboró con este comité en la organización de las Conferencias CIAEM y en la dirección de la temática de Resolución de Problemas. Fue además, miembro del Sistema Nacional de Investigadores, de la Academia Mexicana de Ciencia y de la Academia de Ingeniería de México. En esta última institución coordinó el área de Educación en Ingeniería. Además, fue coordinadora de la Red Internacional de Investigación MaCiCiencias y miembro del Comité Editorial de las revistas Innovación Educativa y Portulano.

Su paso por el Comité Editorial de la Revista Educación Matemática fue breve debido a las múltiples exigencias que tenía en su trabajo como administradora, profesora e investigadora en el Instituto Politécnico Nacional. Pero fue 
siempre generosa en la dedicación de su tiempo a la revisión científica de artículos recibidos para publicación en esta revista.

Patricia nos deja una lección de compromiso y optimismo. La comunidad de investigación en Educación Matemática pierde a una investigadora reconocida por su gentileza y por la calidad de sus trabajos. Nos deja un ejemplo y un trabajo que seguirán siendo fuente de inspiración para la formación de nuevos investigadores. Yo la recordaré siempre como una colega destacada y dedicada, pero más como una amiga alegre y cariñosa. Todos la extrañaremos y la recordaremos siempre.

\section{REFERENCIAS}

Camarena, P. (2013). A treinta años de la teoría educativa "Matemática en el contexto de las ciencias". Innovación Educativa. 13(62), 17-44. 